\title{
A Multiple Power Reachable Sliding Mode Control Approach for Guidance of Miniature Laser Beam Riding Steered Munition
}

\author{
Jun-Fang Fan (iD) and Shi-Wei Chen $(1)$ \\ Beijing Key Laboratory of High Dynamic Navigation Technology, Beijing Information Science \& Technology University, \\ Beijing 100192, China \\ Correspondence should be addressed to Jun-Fang Fan; wyhffjf@bistu.edu.cn
}

Received 23 February 2021; Revised 18 April 2021; Accepted 15 May 2021; Published 30 May 2021

Academic Editor: Juan Du

Copyright (c) 2021 Jun-Fang Fan and Shi-Wei Chen. This is an open access article distributed under the Creative Commons Attribution License, which permits unrestricted use, distribution, and reproduction in any medium, provided the original work is properly cited.

\begin{abstract}
For a two-stage launch miniature shoulder munition steered with a laser beam riding system, the laser beam points to the target with a limited field radius and approximate straight-line spatial path; thus, the munition could not fly into the laser information field after the powered flight. The miniature munition dynamics are established firstly; then, an adaptive multiple power reachable sliding mode controller is presented and adopted to constrain both the trajectory inclination and pitch angle, which makes the munition enter the field and fly under control in the field with desired attitude angles, respectively. Considering the constraints of the incidence angle and the radius of the laser information field, an arctangent function curve is selected as the expected trajectory, and an adaptive multiple power reachable integral sliding mode guidance law is detailed, which makes the munition trajectory approach and converges to the expected curve fastly with limited acceleration. Therefore, the miniature munition flight trajectory is planned and optimized. Convergence and stability are analyzed based on the Lyapunov method. The numerical simulation against the stationary target is performed to fully demonstrate the efficacy of the proposed method.
\end{abstract}

\section{Introduction}

In recent years, infantry shoulder munitions are becoming smaller and lighter with the development of advanced materials and MEMS technology. In order to meet the need for urban and close combats in complex environments, various types of lightweight guided munitions have been put forward, such as Spike (U.S. Navy), Pike (Raytheon), and Spike SR (Rafael). These munitions adopt strapdown electrooptical seeker for target acquisition and guidance and enable forces to shoot-and-scoot without exposing their location. The background munition in this paper is different and adopts a laser beam riding steered (LBRS) system, which has more high guidance precision and better antijamming capability [1]. The essence of the LBRS guidance is to measure the position errors of the munition relative to the central point of the laser information field and generate command to control the munition flying along the centerline of the information field [2]. Usually, the munition should be powered during the flight envelope to maintain a stable altitude, which brings a great challenge to the miniature munition that will not enter the field during the initial two-launch stage. The typical guidance mechanism for the beam riding system generally adopts a three-point method, which has the disadvantage that the miss distance will increase because of the lag of measurement deviation error.

Aiming at the uncertainty in the guidance system, the state feedback control technology is presented in [3], and a controller based on global sliding mode control is proposed in [4] to improve the robustness and stability. Aiming at the synchronization problem of uncertain nonlinear systems, a composite nonlinear feedback control method is detailed in [5], and an adaptive global terminal sliding mode control scheme is shown in [6]. Sliding mode control- (SMC-) based guidance and control design methods can be seen in literature for its good performance in response and robustness to parameter change and disturbance $[7,8]$. For intercepting a nonmaneuvering target, an optimal sliding mode guidance law is proposed combining optimal theory in [9]. For the impact angle constraint problem, an impact angle control 
guidance law, a finite-time convergent SMC guidance law, and guidance law considering both impact time and impact angle constraints are presented in [10-14], respectively.

In order to track the expected line-of-sight rate with uncertainty, a robust second-order SMC combined with the backstepping method is designed in [15]. A guidance law for intercepting a constant-velocity target with a desired impact angle is proposed in [16]. In [17], a guidance law based on SMC is used to intercept stationary, uniform, and maneuvering targets at the desired impact angle. In order to design an impact angle constraint terminal guidance law to improve the warhead effect of air-to-ground guided weapons in [18], a variable structure guidance law with pitch/yaw angle constraints is derived, and saturation function is introduced in the reachable guidance law to weaken the system chattering. In [19], a design scheme for integrated guidance and control (IGC) with terminal impact angle constraint based on SMC is proposed. In [20], a continuous robust impact angle constraint guidance law with finite-time convergence is adopted for maneuvering targets with unknown acceleration boundary. In [21], the SMC-based guidance law is used to adaptively control the unknown uncertainty boundary. In [22], an advanced guidance law based on the nonsingular fast terminal SMC scheme and adaptive control is analyzed. To solve the terminal interception of a maneuvering target, an adaptive integral SMC (ISMC) guidance law and the case considering terminal angle constraint are detailed in $[23,24]$. Aiming at the high precise guidance problem of a hypersonic vehicle in the gliding phase, considering the complex multiconstraints and uncertainty, a guidance law based on global ISMC is discussed in [25].

Due to the radius limitation of the laser information field and the upper arc trajectory during two-stage launch, the miniature munition with LBRS guidance cannot fly into the laser information field directly at the initial flight stage; thus, the traditional three-point guidance mode is not applicable. In this paper, to overcome the limitation of the traditional LBRS guidance mechanism, based on the above research results, a novel guidance law combined SMC with adaptive multiple power reachable (AMPR) method is proposed to constrain the ballistic inclination and pitch angle and make the munition fly into the laser information field with desired attitude angles. Then, the ISMC with AMPR method is presented to shape the trajectory and approach to the desired curve. The convergence and stability are proved based on the Lyapunov stability theorem.

The remainder of this paper is organized as follows. In the second part, the munition dynamics and the expected flight trajectory are described. In the third part, the piecewise guidance law and the control command based on the adaptive multiple power reachable method and SMC method is detailed. In the fourth part, the stability analysis of the proposed guidance law is performed using the Lyapunov theorem. The simulation results and analysis are discussed in the fifth part, and the conclusion is given finally.

\section{Miniature Munition Dynamics and Model}

The conventional guidance strategy of the LBRS system is a three-point method; that is, the launch point, munition, and target are always kept in a straight line [26], but it is difficult to achieve for the miniature munition detailed in this paper. Considering the flight trajectory constraint, the flight inclination angle and pitch angle are selected and combined with SMC to design and optimize the guidance law for the whole flight process. The geometric relations for munition, target, and trajectory are described in Figure 1.

2.1. Start-Up and Control Conditions. According to Figure 1, the control of miniature LBRS munition is divided into two stages.

In the first stage, the judgment conditions are

$$
\begin{aligned}
\frac{d y}{d t} & =0, \\
y & >\Delta r_{m}+\Delta y_{m},
\end{aligned}
$$

where $\Delta r_{m}$ is the effective radius of the laser information field and $\Delta y_{m}$ is the centerline height of the laser beam.

In the second stage, the guided munition flies into the laser beam and toward the target, and the judgment conditions are

$$
\begin{aligned}
\frac{d y}{d t}<0, \\
\Delta y_{m}<y<\Delta r_{m}+\Delta y_{m} .
\end{aligned}
$$

2.2. Munition Dynamics. The coordinate and attitude of a theoretical miniature LRBS munition model are shown in Figure 2, where the coordinate origin is set in the center of the munition gravity, $O X Y Z$ is the inertial coordinate, $O X_{1}$ $Y_{1} Z_{1}$ is the ballistic coordinate, $O X_{2} Y_{2} Z_{2}$ is the munition body coordinate, and $\mathrm{OX}_{3} Y_{3} Z_{3}$ is the flight velocity coordinate; all obey the right-hand rule. The typical diameter and length of this model are $40 \mathrm{~mm}$ and $550 \mathrm{~mm}$, respectively.

The translational dynamics of the munition can be expressed as

$$
\left\{\begin{array}{l}
m \frac{d V}{d t}=P \cos \alpha \cos \beta-X-m g \sin \theta_{m} \\
m V \frac{d \theta_{m}}{d t}=P\left(\sin \alpha \cos \gamma_{V}+\cos \alpha \sin \beta \sin \gamma_{V}\right)+Y \cos \gamma_{V}-Z \sin \gamma_{V}-m g \cos \theta_{m} \\
-m V \cos \theta_{m} \frac{d \psi_{V}}{d t}=P\left(\sin \alpha \sin \gamma_{V}-\cos \alpha \sin \beta \cos \gamma_{V}\right)+Y \sin \gamma_{V}+Z \cos \gamma_{V}
\end{array}\right.
$$




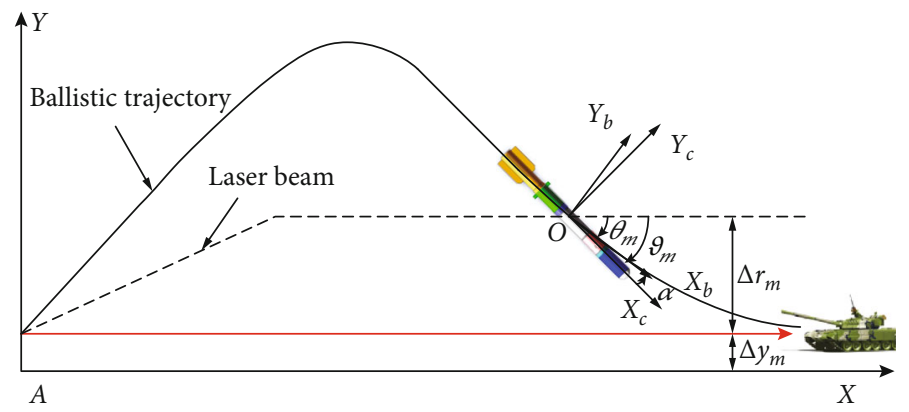

FIgURE 1: Relations of munition, target, and trajectory.

where $\alpha$ is the angle of attack (AoA), $\beta$ is the sideslip angle, $\theta_{m}$ is the ballistic inclination angle, $\psi_{V}$ is the ballistic declination angle, and $\gamma_{V}$ is the velocity inclination angle. The drag $X$, lift $Y$, and lateral force $Z$ can be written as

$$
\begin{aligned}
& X=\frac{1}{2} \rho V^{2} c_{x} S, \\
& Y=\frac{1}{2} \rho V^{2}\left(c_{y}^{\alpha} \alpha+c_{y}^{\delta_{y}} \delta_{z}\right) S, \\
& Z=\frac{1}{2} \rho V^{2}\left(c_{z}^{\beta} \beta+c_{z}^{\delta y} \delta_{y}\right) S .
\end{aligned}
$$

The rotational dynamics of the munition can be written as

$$
\left\{\begin{array}{l}
\frac{d \omega_{x}}{d t}=\frac{1}{J_{x}}\left[-\left(J_{z}-J_{y}\right) \omega_{z} \omega_{y}+M_{x}\right], \\
\frac{d \omega_{y}}{d t}=\frac{1}{J_{y}}\left[-\left(J_{x}-J_{z}\right) \omega_{x} \omega_{z}+M_{y}\right], \\
\frac{d \omega_{z}}{d t}=\frac{1}{J_{z}}\left[-\left(J_{y}-J_{x}\right) \omega_{y} \omega_{x}+M_{z}\right],
\end{array}\right.
$$

where

$$
\begin{aligned}
& M_{x}=\frac{1}{2} \rho V^{2} S L\left(m_{x}^{\bar{\omega}_{x}} \bar{\omega}_{x}+m_{x}^{\delta_{x}} \delta_{x}\right)+\Delta M_{x}, \\
& M_{y}=\frac{1}{2} \rho V^{2} S L\left(m_{y}^{\beta} \beta+m_{y}^{\bar{\omega}_{y}} \bar{\omega}_{y}+m_{y}^{\delta_{y}} \delta_{y}\right)+\Delta M_{y}, \\
& M_{z}=\frac{1}{2} \rho V^{2} S L\left(m_{z}^{\alpha} \alpha+m_{z}^{\bar{\omega}_{z}} \bar{\omega}_{z}+m_{z}^{\delta_{z}} \delta_{z}\right)+\Delta M_{z}, \\
& \bar{\omega}_{x}=\frac{\omega_{x} L}{2 V}, \bar{\omega}_{y}=\frac{\omega_{y} L}{V}, \bar{\omega}_{z}=\frac{\omega_{z} L}{V} .
\end{aligned}
$$

Derivation of Equation (8) gives

$$
\left\{\begin{array}{l}
\ddot{x}_{m}=\dot{V} \cos \theta_{m} \cos \psi_{V}-V \dot{\theta}_{m} \sin \theta_{m} \cos \psi_{V}-V \dot{\psi}_{V} \cos \theta_{m} \sin \psi_{V}, \\
\ddot{y}_{m}=\dot{V} \sin \theta_{m}+V \dot{\theta}_{m} \cos \theta_{m}, \\
\ddot{z}_{m}=-\dot{V} \cos \theta_{m} \sin \psi_{V}+V \dot{\theta}_{m} \sin \theta_{m} \sin \psi_{V}-V \dot{\psi}_{V} \cos \theta_{m} \cos \psi_{V} .
\end{array}\right.
$$

The first- and second-order derivatives for the inclination angle can be obtained from Equation (3):

$$
\begin{gathered}
\dot{\theta}_{m}=\frac{P\left(\sin \alpha \cos \gamma_{V}+\cos \alpha \sin \beta \sin \gamma_{V}\right)+Y \cos \gamma_{V}-Z \sin \gamma_{V}-m g \cos \theta_{m}}{m V}, \\
\ddot{\theta}_{m}=\frac{P\left[\dot{\alpha}\left(\cos \alpha \cos \gamma_{V}-\sin \alpha \sin \beta \sin \gamma_{V}\right)-\dot{\gamma}_{V}\left(\sin \alpha \sin \gamma_{V}-\cos \alpha \sin \beta \cos \gamma_{V}\right)\right]}{m V} \\
+\frac{P \dot{\beta} \cos \alpha \cos \beta \sin \gamma_{V}+\dot{Y} \cos \gamma_{V}-Y \dot{\gamma}_{V} \sin \gamma_{V}-\dot{Z} \sin \gamma_{V}-Z \dot{\gamma}_{V} \cos \gamma_{V}+\dot{\theta}_{m} m g \sin \theta_{m}}{m V} \\
-\frac{\left[P\left(\sin \alpha \cos \gamma_{V}+\cos \alpha \sin \beta \sin \gamma_{V}\right)+Y \cos \gamma_{V}-Z \sin \gamma_{V}-m g \cos \theta_{m}\right] \dot{V}}{m V^{2}} .
\end{gathered}
$$




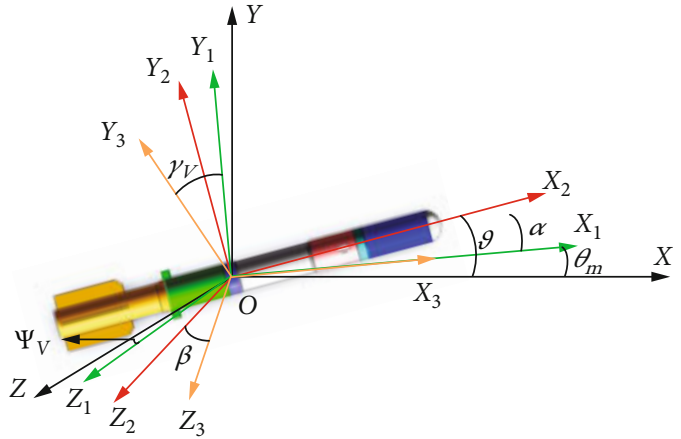

FIgURE 2: Coordinate and attitude of a miniature LBRS munition.

Substituting Equation (4) into Equation (11):

$$
\ddot{\theta}_{m}=a_{c} \delta_{z}+b_{c} \delta_{y}+f_{c} \text {, }
$$

where

$$
\begin{aligned}
& a_{c}=\frac{-q S c_{y}^{\delta_{z}}}{m V^{2}}\left(\dot{\gamma}_{V} V \sin \gamma_{V}+\dot{V} \cos \gamma_{V}\right), \\
& b_{c}=\frac{q S c_{z}^{\delta_{y}}}{m V^{2}}\left(-\dot{\gamma}_{V} V \cos \gamma_{V}+\dot{V} \sin \gamma_{V}\right), \\
& f_{c}=\frac{P\left(\mathrm{~A}_{1}+\dot{\beta} \cos \alpha \cos \beta \sin \gamma_{V}\right)+\mathrm{A}_{2}}{m V}-\frac{\mathrm{A}_{3} \dot{V}}{m V^{2}},
\end{aligned}
$$

where

$$
\begin{aligned}
\mathrm{A}_{1}= & \dot{\alpha}\left(\cos \alpha \cos \gamma_{V}-\sin \alpha \sin \beta \sin \gamma_{V}\right) \\
& -\dot{\gamma}_{V}\left(\sin \alpha \sin \gamma_{V}-\cos \alpha \sin \beta \cos \gamma_{V}\right), \\
\mathrm{A}_{2}= & \dot{Y} \cos \gamma_{V}-c_{y}^{\alpha} \alpha q S \dot{\gamma}_{V} \sin \gamma_{V}-\dot{Z} \sin \gamma_{V} \\
& -c_{z}^{\beta} \beta q S \dot{\gamma}_{V} \cos \gamma_{V}+\dot{\theta}_{m} m g \sin \theta_{m}, \\
\mathrm{~A}_{3}= & P\left(\sin \alpha \cos \gamma_{V}+\cos \alpha \sin \beta \sin \gamma_{V}\right)+c_{y}^{\alpha} \alpha q S \cos \gamma_{V} \\
& -c_{z}^{\beta} \beta q S \sin \gamma_{V}-m g \cos \theta_{m} .
\end{aligned}
$$

Similarly, the pitch angle derivatives can be obtained from Equation (7):

$$
\dot{\vartheta}_{m}=\omega_{y} \sin \gamma+\omega_{z} \cos \gamma,
$$

$$
\ddot{\vartheta}_{m}=\dot{\omega}_{y} \sin \gamma+\dot{\gamma} \omega_{y} \cos \gamma+\dot{\omega}_{z} \cos \gamma-\dot{\gamma} \omega_{z} \sin \gamma .
$$

Substituting both Equations (5) and (6) into Equation (16) yields

$$
\ddot{\vartheta}_{m}=a_{m} \delta_{z}+b_{m} \delta_{y}+f_{m},
$$

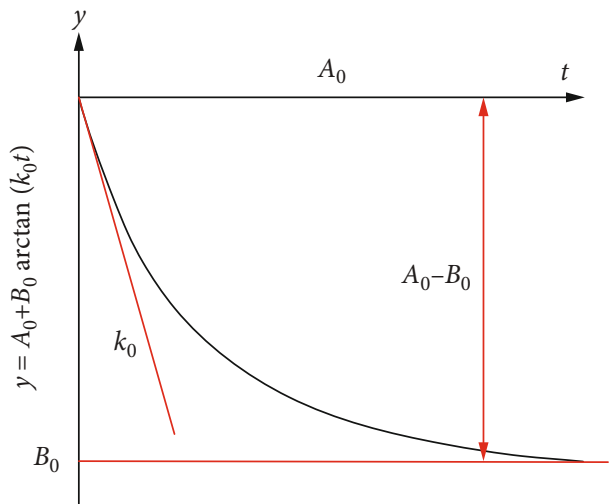

FIGURE 3: Arctangent function curve.

where

$$
\begin{aligned}
a_{m}= & \frac{q S L m_{z}^{\delta_{z}} \cos \gamma}{J_{z}}, \\
b_{m}= & \frac{q S L m_{y}^{\delta_{y}} \sin \gamma}{J_{y}}, \\
f_{m}= & \frac{-\left(J_{x}-J_{z}\right) \omega_{x} \omega_{z}+q S L\left(m_{y}^{\beta} \beta+m_{y}^{\bar{\omega}_{y}} \bar{\omega}_{y}\right)}{J_{y}} \sin \gamma \\
& +\frac{-\left(J_{y}-J_{x}\right) \omega_{y} \omega_{x}+q S L\left(m_{z}^{\alpha} \alpha+m_{z}^{\bar{\omega}_{z}} \bar{\omega}_{z}\right)}{J_{z}} \cos \gamma \\
& +\dot{\gamma}\left(\omega_{y} \cos \gamma-\omega_{z} \sin \gamma\right) .
\end{aligned}
$$

2.3. Expected Trajectory Model. Inspiring from Figure 3, an arctangent function is proposed with the form as

$$
y=A_{0}+B_{0} \arctan \left(k_{0} t\right) .
$$

This curve decreases rapidly and tends to be gentle with time, where the intersection with the longitudinal axis, the descending height, and the slope of the zero point of the coordinate are determined by $A_{0}, B_{0}$, and $k_{0}$, respectively. Thus, the shape of the curve can be shaped using different parameter sets, and the part where the positive transverse axis of arctangent function is a well choice for the terminal trajectory of the miniature LBRS munition.

The expected trajectory curve has the form below after the munition enters the laser beam:

$$
y_{n}=y_{0}+k_{n 1} \arctan \left(k_{n 2} x_{n}\right) \text {, }
$$

where $y_{0}=\Delta y_{m}+\Delta r_{m}$ is the initial height, $k_{n 1}=-\Delta r_{m}$ is the final height to be lowered, $k_{n 2}=\theta_{n} \pi / 180$ is the initial trajectory inclination angle, and $x_{n}=x_{m}$ is the transverse position. 
The derivatives of Equation (20) can be calculated as follows:

$$
\begin{aligned}
& \dot{y}_{n}=\frac{k_{n 1} k_{n 2}}{1+\left(k_{n 2} x_{n}\right)^{2}}, \\
& \ddot{y}_{n}=\frac{-2 k_{n 1} k_{n 2}^{3} x_{n} \dot{x}_{n}}{\left[1+\left(k_{n 2} x_{n}\right)^{2}\right]^{2}} .
\end{aligned}
$$

\section{Guidance Law Design}

The adaptive multiple power reachable (AMPR) method proportional differential SMC is used to constrain the angle, and the adaptive multiple power reachable integral sliding mode guidance law (AMPR-ISMGL) is used to constrain the trajectory.

3.1. Angle Constraint SMC Guidance Law. In order to make the LBRS munition fly into the laser information field at a constant angle, the inclination angle $\theta$ should be constrained, while the pitch angle $\vartheta$ should be also limited for small AoA. Thus, select $\vartheta_{n}$ and $\theta_{n}$ as the constraint.

Inspired by Reference [27], the sliding mode surface $S$ $=\left[s_{1}, s_{2}\right]$ is selected as

$$
\left\{\begin{array}{l}
s_{1}=c_{1}\left(\vartheta_{m}-\vartheta_{n}\right)+\left(\dot{\vartheta}_{m}-\dot{\vartheta}_{n}\right), \\
s_{2}=c_{2}\left(\theta_{m}-\theta_{n}\right)+\left(\dot{\theta}_{m}-\dot{\theta}_{n}\right),
\end{array}\right.
$$

where $c_{1}, c_{2}>0$ for adjusting the state approaching speed, which can be determined according to the pole arrangement on the premise that the state dynamic characteristics are far less than the control loop dynamic characteristics.

The derivation expression has the form below:

$$
\left\{\begin{array}{l}
\dot{s}_{1}=c_{1}\left(\dot{\vartheta}_{m}-\dot{\vartheta}_{n}\right)+\left(\ddot{\vartheta}_{m}-\ddot{\vartheta}_{n}\right), \\
\dot{s}_{2}=c_{2}\left(\dot{\theta}_{m}-\dot{\theta}_{n}\right)+\left(\ddot{\theta}_{m}-\ddot{\theta}_{n}\right) .
\end{array}\right.
$$

To avoid the chattering, slow convergence, and unsmooth response of the traditional SMC method, an AMPR guidance law is proposed as follows:

$$
\begin{aligned}
\dot{s}= & -k_{1}|s|^{\alpha_{r}} \operatorname{sgn} s-k_{2}\left(\frac{1}{|s|+1}\right)|s|^{\beta_{r}} \operatorname{sgn} s-k_{3}|s|^{\gamma_{r}} \operatorname{sgn} s-k_{4} \\
& \cdot\left(\frac{1}{|s|+0.1}\right) s
\end{aligned}
$$

where $k_{i}>0, i=1,2,3,4, \alpha_{r}>1,0<\beta_{r}<1$, and the value of $\gamma_{r}$ is

$$
\gamma_{r}= \begin{cases}\max \left\{\alpha_{r},|s|\right\}, & |s| \geq 1 \\ \min \left\{\beta_{r},|s|\right\}, & |s|<1\end{cases}
$$

TABLE 1: Adaptive multiple power reachable SMC guidance law parameters.

\begin{tabular}{cccccccccccc}
\hline$k_{1}$ & $k_{2}$ & $k_{3}$ & $k_{4}$ & $\alpha_{r}$ & $\beta_{r}$ & $c_{1}$ & $c_{2}$ & $c_{3}$ & $c_{4}$ & $\alpha_{e 1}$ & $\alpha_{e 2}$ \\
\hline 5 & 3 & 5 & 4 & 1.5 & 0.5 & 0.7 & 0.8 & 0.5 & 0.8 & 0.5 & 0.75 \\
\hline
\end{tabular}

Due to the performance of the exponential function, Equation (24) is mainly affected by $-k_{1}|s|^{\alpha_{r}} \operatorname{sgn} s-k_{3}|s|^{\gamma_{r}}$ sgn $s$ and $-k_{2}[1 /(|s|+1)]|s|^{\beta_{r}}$ sgn $s-k_{3}|s|^{\gamma_{r}}$ sgn $s$ when the system state satisfies $|s| \geq 1$ and $|s|<1$, respectively. When $|s|$ is very small, Equation (24) is mainly determined by $-k_{4}$ $[1 /(|s|+0.1)] s . \gamma_{r}$ in Equation (25) ensures that the system adaptively changes the exponential parameters once the state satisfies $|s|>\alpha_{r}$ or $|s|<\beta_{r}$, thus yielding a faster convergence rate.

Then, the corresponding fin deflection commands can be calculated by Equation (23) and (24):

$$
\begin{gathered}
\delta_{z c}=-\frac{1}{a_{c}}\left(c_{2} \dot{\theta}_{m}+b_{c} \delta_{y}+f_{c}-\mathrm{B}\right), \\
\delta_{z m}=-\frac{1}{a_{m}}\left(c_{1} \dot{\vartheta}_{m}+b_{m} \delta_{y}+f_{m}-\mathrm{B}\right),
\end{gathered}
$$

where

$$
\mathrm{B}=-\operatorname{sgn} s\left[k_{1}|s|^{\alpha_{r}}+k_{2}\left(\frac{1}{|s|+1}\right)|s|^{\beta_{r}}+k_{3}|s|^{\gamma_{r}}\right]-k_{4}\left(\frac{1}{|s|+0.1}\right) s .
$$

When the munition enters the laser beam, let $\vartheta_{n 1}$ be equal to $\theta_{n 1}$ to suppress the AoA $\alpha$ :

$$
\theta_{n 1}=\vartheta_{n 1}=\arctan \left(\frac{d y_{n}}{d x_{n}}\right)
$$

Likewise, the deflection commands $\delta_{z c 1}$ and $\delta_{z m 1}$ can be calculated as

$$
\begin{aligned}
& \delta_{z c 1}=-\frac{1}{a_{c}}\left[c_{2}\left(\dot{\theta}_{m}-\dot{\theta}_{n 1}\right)+\left(b_{c} \delta_{y}+f_{c}-\ddot{\theta}_{n 1}\right)-B\right], \\
& \delta_{z m 1}=-\frac{1}{a_{m}}\left[c_{1}\left(\dot{\vartheta}_{m}-\dot{\vartheta}_{n 1}\right)+\left(b_{m} \delta_{y}+f_{m}-\ddot{\vartheta}_{n 1}\right)-B\right],
\end{aligned}
$$

where $\dot{\theta}_{n 1}$ and $\ddot{\theta}_{n 1}$ have the form as follows:

$$
\begin{aligned}
& \dot{\theta}_{n 1}=-\frac{2 k_{n 1} k_{n 2}^{3} x_{n} \dot{x}_{n}^{2}+D k_{n 1} k_{n 2} \ddot{x}_{n}}{D^{2} \dot{x}_{n}^{2}\left[1+\left(k_{n 1} k_{n 2} / D \dot{x}_{n}\right)^{2}\right]}, \\
& \ddot{\theta}_{n 1}=\frac{-\mathrm{K}_{1} \mathrm{~K}_{2}+\mathrm{K}_{3}\left(2 k_{n 1} k_{n 2}^{3} x_{n} \dot{x}_{n}^{2}+D k_{n 1} k_{n 2} \ddot{x}_{n}\right)}{K_{1}^{2}},
\end{aligned}
$$


TABLE 2: Initial munition data.

\begin{tabular}{lccccc}
\hline Initial position & Initial velocity & Launch angle & Laser beam radius & Height of centerline & Constraint angle \\
\hline$P_{m 0}=\left[\begin{array}{ll}0 & 1\end{array}\right]^{T}$ & $V_{0}=\left[\begin{array}{ll}29.4 & 6\end{array}\right]^{T}$ & $\vartheta_{0}=11.5(\mathrm{deg})$ & $\Delta r_{m}=8(\mathrm{~m})$ & $\Delta y_{m}=1(\mathrm{~m})$ & $\vartheta_{n}=\theta_{n}=-2(\mathrm{deg})$ \\
\hline
\end{tabular}

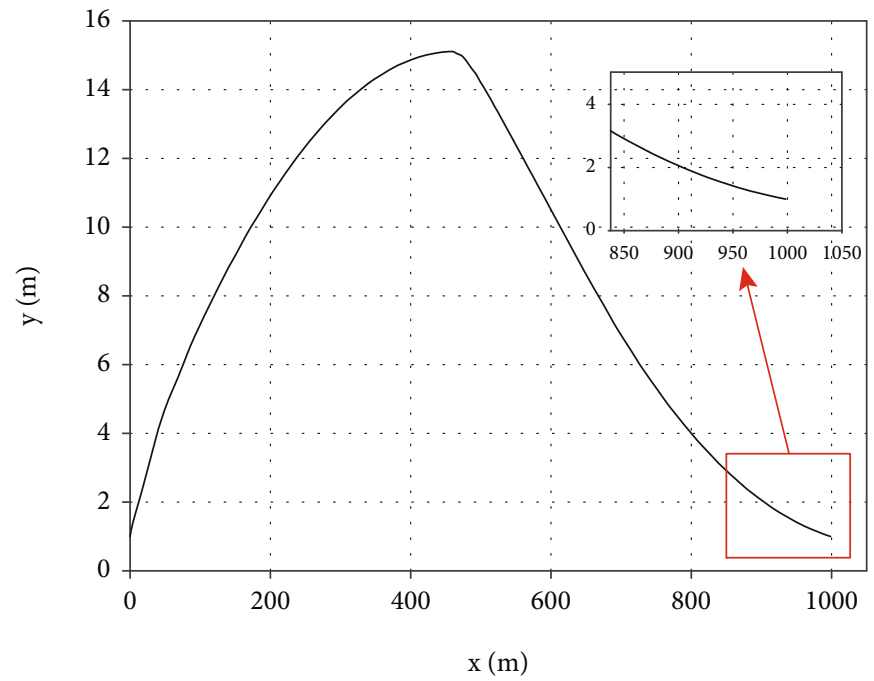

FIgURE 4: Flight trajectory.
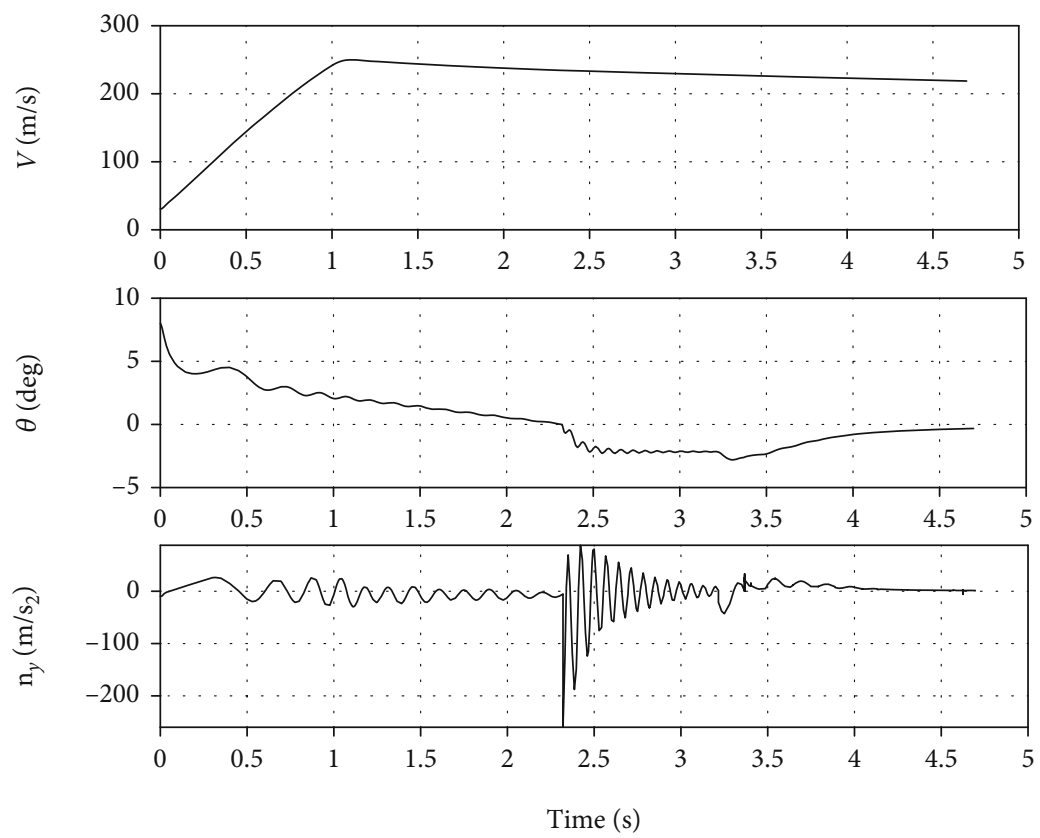

Figure 5: Velocity, inclination angle, and acceleration.

where $\mathrm{K}_{1}=D^{2} x_{n}^{2}\left[1+\left(k_{n 1} k_{n 2} / D x_{n}\right)^{2}\right], \quad \mathrm{K}_{2}=2 k_{n 1} k_{n 2}^{2} x_{n}\left(k_{n 2}\right.$ $\left.x_{n}^{2}+2 k_{n 2} x_{n} x_{n}{ }_{n}+x_{n} x^{*}{ }_{n}\right), \mathrm{K}_{3}=2 x_{n}{ }_{n} x_{n} D^{2}\left[1+\left(k_{n 1} k_{n 2} / D x_{n}\right)^{2}\right]$ $-2\left(k_{n 1} k_{n 2}\right)^{2}\left(2 k_{n 2} x_{n} x_{n}^{2}+D x_{n}{ }_{n}\right)$, and $D=1+\left(k_{n 2} x_{n}\right)^{2}$.

3.2. Trajectory-Keeping SMC Guidance Law. The fundamental control strategy of miniature LBRS munition is to use the AMPR-ISMGL to restrain the trajectory to approach the centerline of laser beam quickly and fly along the centerline until hitting the target.
Select the state variables $x_{e 1}=y_{m}-y_{n}$ and $x_{e 2}=x_{e 1}$ with state equation:

$$
\left\{\begin{array}{l}
\dot{x}_{e 1}=x_{e 2}, \\
\dot{x}_{e 2}=\dot{V} \sin \theta_{m}+V \dot{\theta}_{m} \cos \theta_{m}+\frac{2 k_{n 1} k_{n 2}^{3} x_{n} \dot{x}_{n}}{\left[1+\left(k_{n 2} x_{n}\right)^{2}\right]^{2}}
\end{array}\right.
$$



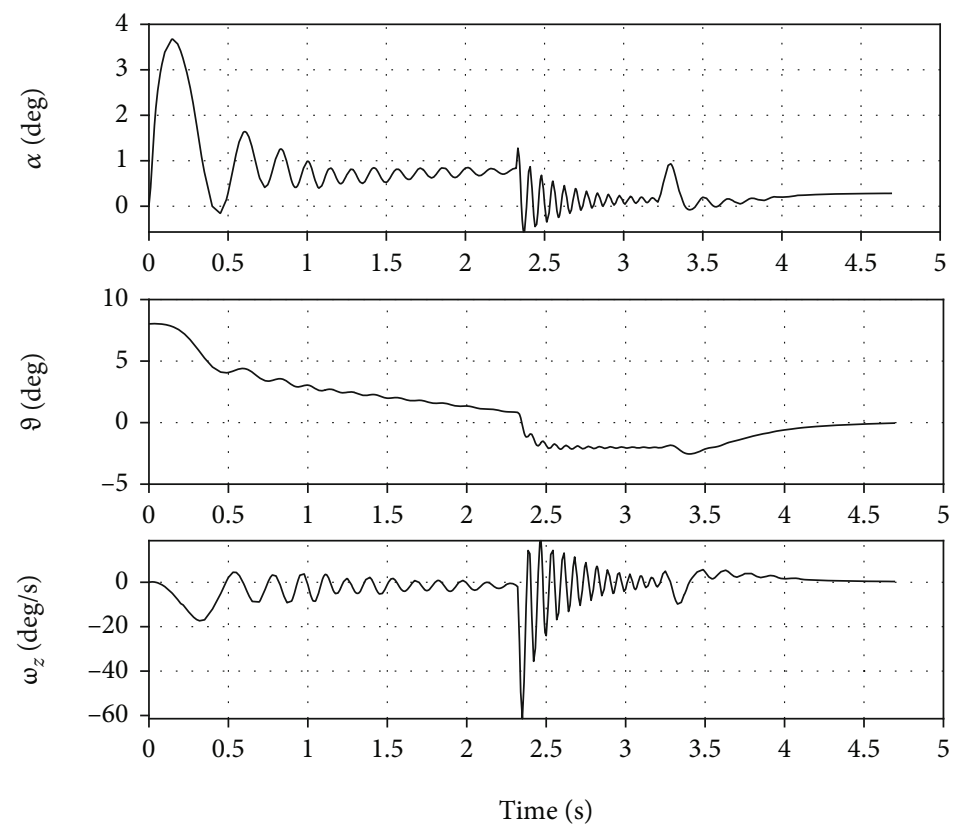

FIgURe 6: AoA, pitch angle, and pitch rate.
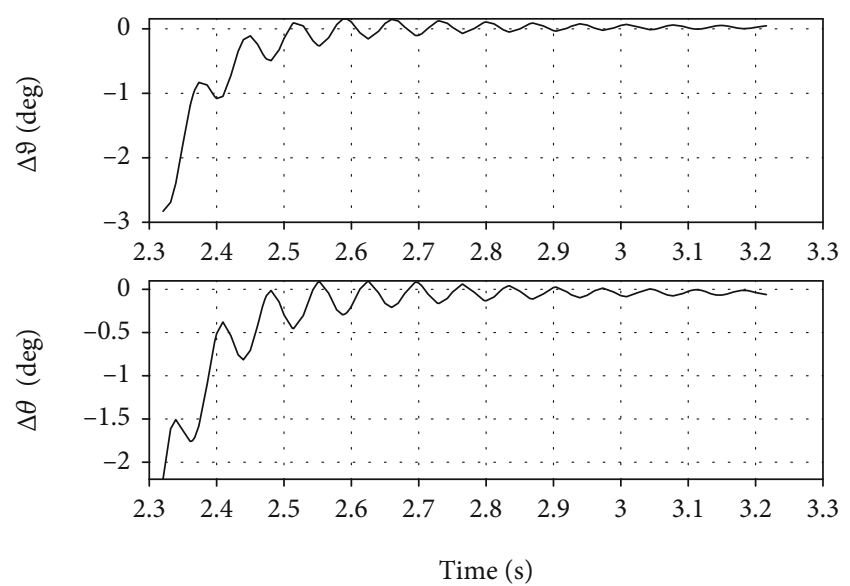

FIGURE 7: Deviation curve of pitch angle and inclination angle.

Then, the integral sliding mode surface is selected as

$$
s_{3}=x_{e 2}+\int_{0}^{t}\left(c_{3}\left|x_{e 1}\right|^{\alpha_{e 1}} \operatorname{sgn} x_{e 1}+c_{4}\left|x_{e 2}\right|^{\alpha_{e 2}} \operatorname{sgn} x_{e 2}\right) d t .
$$

According to [28], if $c_{3}, c_{4}>0$ satisfy the Hurwitz stability criterion, the state feedback control $c_{3}\left|x_{e 1}\right|^{\alpha_{e 1}} \operatorname{sgn} x_{e 1}+c_{4}$ $\left|x_{e 2}\right|^{\alpha_{e 2}} \operatorname{sgn} x_{e 2}$ in Equation (33) can stabilize the system for finite time, and $\alpha_{e 1}, \alpha_{e 2}$ satisfy $\alpha_{e 1}=\alpha_{e 2} \alpha_{e 3} /\left(2 \alpha_{e 3}-\alpha_{e 2}\right), \alpha_{e 2}$ $=\alpha, \alpha_{e 3}=1, \varepsilon \in(0,1), \alpha \in(1-\varepsilon, 1)$.

Derivation of Equation (33) gives

$$
\dot{s}_{3}=\dot{x}_{e 2}+c_{3}\left|x_{e 1}\right|^{\alpha_{e 1}} \operatorname{sgn} x_{e 1}+c_{4}\left|x_{e 2}\right|^{\alpha_{e 2}} \operatorname{sgn} x_{e 2} .
$$

Substituting Equation (32) into Equation (34) obtains

$$
\begin{aligned}
\dot{s}_{3}= & c_{3}\left|x_{e 1}\right|^{\alpha_{e 1}} \operatorname{sgn} x_{e 1}+c_{4}\left|x_{e 2}\right|^{\alpha_{e 2}} \operatorname{sgn} x_{e 2}+\dot{V} \sin \theta_{m} \\
& +V \dot{\theta}_{m} \cos \theta_{m}+\frac{2 k_{n 1} k_{n 2}^{3} x_{n} \dot{x}_{n}}{\left[1+\left(k_{n 2} x_{n}\right)^{2}\right]^{2}} .
\end{aligned}
$$

Then, the guidance command $a_{n}$ is given as

$$
\begin{aligned}
a_{n}= & -\frac{1}{\sin \theta_{m}}\left\{c_{3}\left|x_{e 1}\right|^{\alpha_{e 1}} \operatorname{sgn} x_{e 1}+c_{4}\left|x_{e 2}\right|^{\alpha_{e 2}} \operatorname{sgn} x_{e 2}+V \dot{\theta}_{m} \cos \theta_{m}\right. \\
& \left.+\frac{2 k_{n 1} k_{n 2}^{3} x_{n} \dot{x}_{n}}{\left[1+\left(k_{n 2} x_{n}\right)^{2}\right]^{2}}-\mathrm{B}\right\} .
\end{aligned}
$$

Considering the practical application, the main variables in Equation (36) can be analyzed as follows. Wherein, rate $\omega_{z}$, velocity $V$, and acceleration $a$ can be directly measured by an inertial measurement unit (IMU). Position information $x_{n}=x_{m}$ and $y_{m}$ can be obtained by IMU navigation integration or measured directly by the onboard laser receiver; $y_{n}$ can be obtained by Equation (20). Similarly, for the variables involved in the angle constraint commands Equations (26) and (29), the ballistic inclination angle $\theta_{m}$ is obtained by the tangent value of the velocity component, and the rudder deflection angle $\delta_{y}$ can be obtained using the feedback data of the rudder system. Besides, the approaching speed is mainly determined by sliding surface coefficients $\alpha_{e i}, i=1,2, c_{i}>0, i=1,2,3,4$, and reaching law coefficients $\alpha_{r}, \beta_{r}, \gamma_{r}$, and $k_{i}>0, i=1,2,3,4$. 


\section{Stability Analysis}

\subsection{Proof of Existence and Accessibility}

Theorem 1. For the proposed guidance law Equation (24), the system state $s$ can reach the equilibrium point $s=0$ under the control action.

Proof. According to the reachable law expression, the following equation holds:

$$
\begin{aligned}
s \dot{s}= & -k_{1}|s|^{\alpha_{r}+1} \operatorname{sgn} s-k_{2}\left(\frac{1}{|s|+1}\right)|s|^{\beta_{r}+1} \operatorname{sgn} s \\
& -k_{3}|s|^{\gamma_{r}+1} \operatorname{sgn} s-k_{4}\left(\frac{1}{|s|+0.1}\right) s^{2} \leq 0 .
\end{aligned}
$$

That is, $s s \leq 0$, if and only if $s=0$, there is $s s=0$.

According to the existence and accessibility conditions of sliding mode reachable law for continuous systems in Ref. [29], if $s \dot{s} \leq 0$ satisfied, the designed control law is existing and reachable; that is, the system state $s$ can reach the equilibrium point $s=0$ under the control law Equation (24).

Lemma 2. Lyapunov stability in finite time [30]. For a continuous nonlinear system,

$$
\dot{x}=f(x), x \in \mathbb{R}^{n},
$$

where $f(0)=0$ and $U$ is an open neighborhood set containing the origin. Given Lyapunov function $V(x): U \rightarrow \mathbb{R}$ and real number $k, \alpha_{r}$ that satisfies $k>0$ and $\alpha_{r} \in(0,1)$, it makes

$$
\dot{V}(x)+k V(x)^{\alpha_{r}} \leq 0
$$

where $V \cdot(x)=\partial V(x) / \partial x f(x)$; then, the origin of the nonlinear system is Lyapunov stable; that is, it is finite-time stable, and the time to reach the origin satisfies $t<V(x(0))^{1-\alpha_{r}} / k\left(1-\alpha_{r}\right)$.

Let the Lyapunov function as $V=1 / 2 s^{2}$ :

$$
\begin{aligned}
\dot{V}= & s \dot{s}=-\operatorname{sgn} s\left[k_{1}|s|^{\alpha_{r}+1}+k_{2}\left(\frac{1}{|s|+1}\right)|s|^{\beta_{r}+1}+k_{3}|s|^{\gamma_{r}+1}\right] \\
& -k_{4}\left(\frac{1}{|s|+0.1}\right) s^{2}<-k_{1}|s|^{\alpha_{r}+1},
\end{aligned}
$$

where $k_{1}, k_{2}, k_{3}$, and $k_{4}$ are all positive, $\alpha_{r}>1,0<\beta_{r}<1$.

$s$ is satisfied regardless of the value of $V \cdot \leq-k_{1}|s|^{\alpha_{r}+1}$. There is a positive constant $Q \in\left(0, k_{1}\right)$ and $\delta \in(0,1)$, so that

$$
\dot{V} \leq-k_{1}\left(s^{2}\right)^{\left(\alpha_{r}+1\right) / 2}=-k_{1}\left(s^{2}\right)^{\delta} \leq-Q\left(s^{2}\right)^{\delta} .
$$

Considering $V=1 / 2 s^{2}$, thus $V^{\cdot}+Q V^{\delta} \leq 0$ is established.

According to Lemma 2, the proposed control law can guarantee the system finite-time stability. In addition,
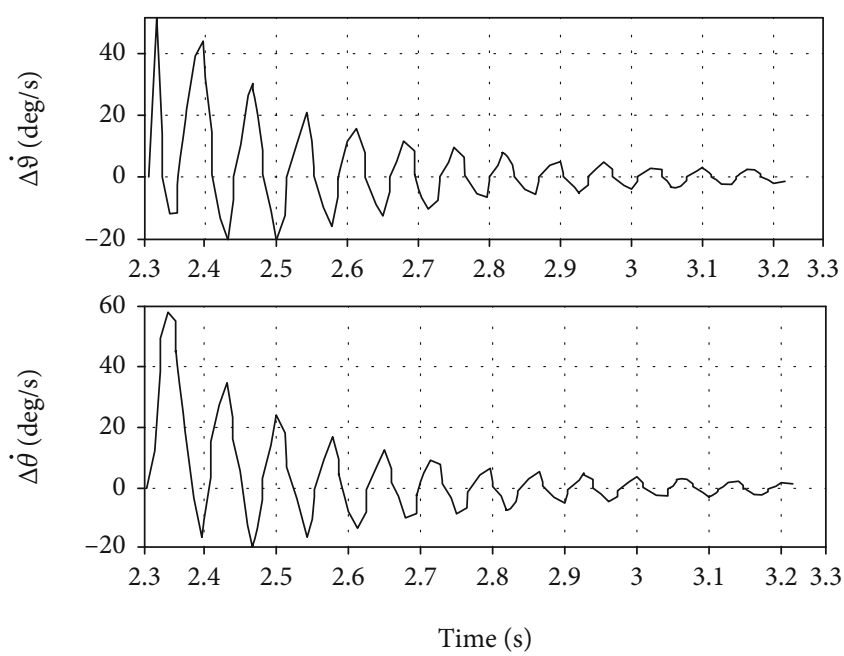

Figure 8: Curve of change rate of pitch angle.

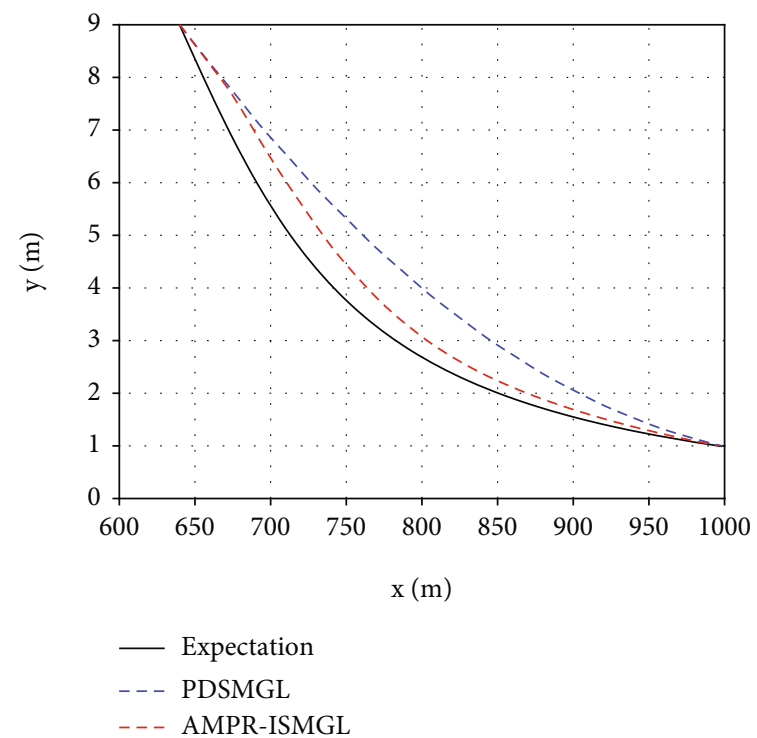

FIgURE 9: Trajectory comparison and trajectory inclination deviation.

according to Theorem 1 , the sliding surface variable $s$ can converge to zero in finite time under the action of the proposed reachable law.

4.2. Stability Analysis of Guidance Law. Based on the aforementioned proof and Lyapunov stability criterion, the solved guidance and control commands can make the designed sliding mode surfaces in Equations (22) and (33) asymptotically stable and further ensure the system converges to stability during finite time in the presence of uncertainty.

Accordingly, it can be seen before the miniature LBRS munition passes through the highest trajectory point and does not enter the laser information field, its attitudes satisfy $\vartheta_{m} \rightarrow \vartheta_{n}, \vartheta_{m} \rightarrow \vartheta_{n}, \theta_{m} \rightarrow \theta_{n}, \theta_{m} \rightarrow \theta_{n}$. Then, when the munition flies in the laser information field until it hits the target, its attitudes satisfy $\vartheta_{m} \rightarrow \vartheta_{n 1}, \vartheta_{m} \rightarrow \vartheta_{n 1}, \theta_{m} \rightarrow \theta_{n 1}$, $\theta_{m} \rightarrow \theta_{n 1}, y_{m} \rightarrow y_{n}$. 

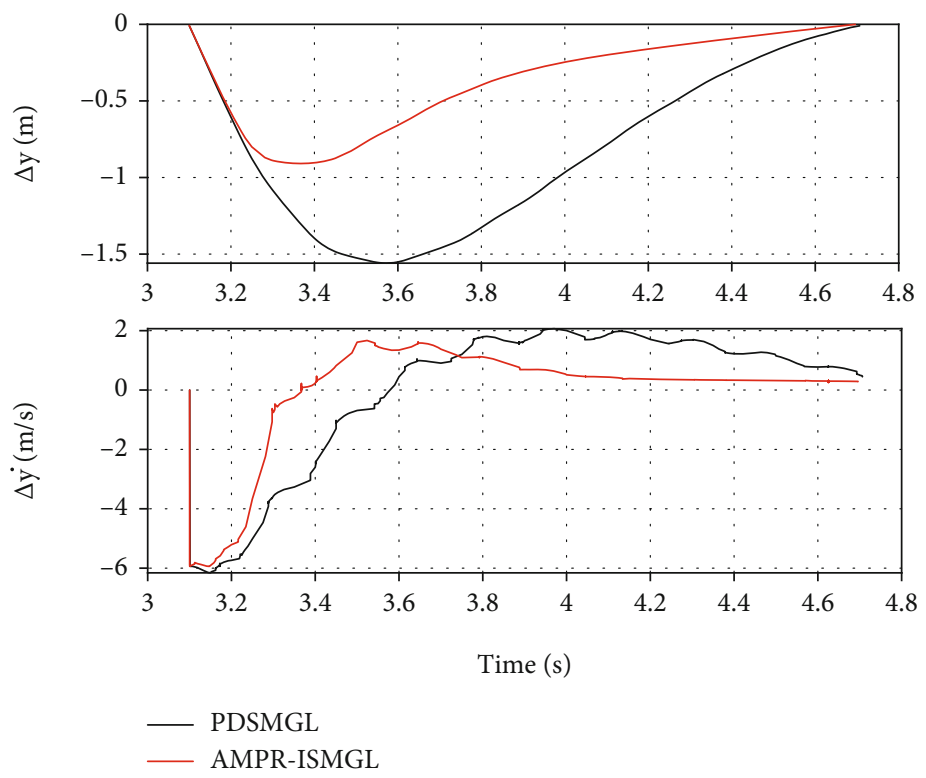

FIgURE 10: Trajectory deviation and deviation rate curve.

\section{Simulation Results and Analysis}

The simulation conditions include the guidance parameters shown in Table 1 and the initial munition flight data shown in Table 2.

The saturation function $\operatorname{sat}(\cdot)$ is used instead of $\operatorname{sgn}(\cdot)$ for smooth consideration:

$$
\operatorname{sat}(s)= \begin{cases}1, & s>\Delta, \\ \frac{s}{\Delta}, & |s| \leq \Delta, \\ -1, & s<-\Delta,\end{cases}
$$

where $\Delta$ is the boundary layer, $\Delta=0.0001$.

The flight trajectory, velocity, trajectory inclination angle, and normal acceleration are shown in Figures 4 and 5. It can be seen that the trajectory inclination angle quickly approaches the set desired value after passing the highest trajectory point, and the normal acceleration converges to zero after a short oscillation.

The AoA, pitch angle, and pitch rate are shown in Figure 6. The AoA of the miniature LBRS munition is small during the unguided stage and converges to zero quickly once the pitch angle is constrained to the set value and the pitch rate converges to zero quickly, which validates the effectiveness of the proposed adaptive multiple power reachable sliding mode guidance law. The curves of pitch angle deviation $\Delta \vartheta=\vartheta_{m}-\vartheta_{n}$ and trajectory inclination deviation $\Delta \theta=\theta_{m}-\theta_{n}$ are shown in Figure 7 .

The curves of pitch angle deviation change rate $\Delta \vartheta$ and trajectory inclination angle deviation change rate $\Delta \theta$ are shown in Figure 8, both of which quickly converge to zero.

In order to objectively evaluate the ballistic trajectory constraint performance of the algorithm in this paper, the proportional differential sliding mode guidance law
(PDSMGL) is also simulated under the same initial conditions. The trajectory comparison curve of guided munition after entering the laser information field is shown in Figure 9. Compared with the traditional PDSMGL, the trajectory using the proposed guidance law in this paper approaches the desired trajectory faster and stably.

The curves of trajectory deviation $\Delta y=y_{m}-y_{n}$ and rate $\Delta y$ between real trajectory and desired trajectory are shown in Figure 10. It can be seen that both methods can ensure that the trajectory error tends to zero. The proposed AMPRISMGL in this paper has a faster response speed for large sliding mode surface error, while the oscillation near the sliding mode surface is smaller.

\section{Conclusion}

In this paper, a multiple-power adaptive reachable guidance law based on the SMC method is proposed for a two-stage launch miniature LBRS munition. The pitch angle and trajectory inclination angle are converged to the expectation value quickly once the munition achieves the highest trajectory point, which guarantees the munition entering the desired laser information field. During flight in the laser information field, the ballistic trajectory with the laser beam radius and the terminal parallel constraint is planned based on the arctangent curve, and an AMPR-ISMGL is detailed to shape the trajectory into the desired curve. The trajectory is smooth with deviation converging to zero at the end; compared with PDSMGL, AMPR-ISMGL has faster convergence speed and smaller oscillation, which proves that the guidance law can effectively restrain trajectory and angle. The existence and accessibility of the proposed guidance method are analyzed using the Lyapunov theorem. The simulation results validate that the presented multiple-power sliding mode guidance law is feasible for the guidance and control of the miniature guided munition. 
To the best of the authors' knowledge, there are few results of complex guidance laws for the LBRS system. Because the guidance and control time of the miniature munition system is very short, subsequent research could be focused on the optimization for guidance parameters and approaching speed, as well as the further hardware-inloop simulation implementation.

\section{Data Availability}

There is no website with available data yet.

\section{Conflicts of Interest}

The authors declare that they have no conflicts of interest.

\section{Acknowledgments}

This work is supported in part by the National Key Research \& Development Program under Grant No. 2020YFC1511705 and the National Natural Science Foundation of China under Grant No. 61801032.

\section{References}

[1] K. B. Wang, "Present situation, key technologies and development of laser guided weapons," Infrared and Laser Engineering, vol. 36, no. 5, pp. 651-655, 2007.

[2] T. F. Zhang, H. X. Zhang, J. J. Hui, F. Meng, and J. J. Qiang, "Development and application of laser guided weapons," Electro-Optic and Control, vol. 22, no. 10, pp. 62-67, 2015.

[3] S. Mobayen and F. Tchier, "Synchronization of a class of uncertain chaotic systems with Lipschitz nonlinearities using state-feedback control design: a matrix inequality approach," Asian Journal of Control, vol. 20, no. 1, pp. 1-15, 2018.

[4] S. Mobayen, "A novel global sliding mode control based on exponential reaching law for a class of underactuated systems with external disturbances," Journal of Computational and Nonlinear Dynamics, vol. 11, no. 2, article 021011, 2015.

[5] S. Mobayen and F. Tchier, "Composite nonlinear feedback control technique for master/slave synchronization of nonlinear systems," Nonlinear Dynamics, vol. 87, no. 3, pp. 17311747, 2017.

[6] M. Saleh, "Adaptive global terminal sliding mode control scheme with improved dynamic surface for uncertain nonlinear systems," International Journal of Control Automation and Systems, vol. 16, no. 2018, pp. 1692-1700, 2018.

[7] Y. Nan, H. X. Chen, Y. Yang, and K. N. Lv, "Research status and prospect of modern main control methods," Journal of Nanjing University of Aeronautics and Astronautics, vol. 47, no. 6, pp. 798-810, 2015.

[8] S. D. Brierley and R. Longchamp, "Application of slidingmode control to air-air interception problem," IEEE Transactions on Aerospace and Electronic Systems, vol. 26, no. 2, pp. 306-325, 1990.

[9] D. Zhou, C. Mu, Q. Ling, and W. Xu, "Optimal sliding-mode guidance of a homing-missile," in Proceedings of the 38th IEEE Conference on Decision and Control (Cat. No. 99CH36304), pp. 5131-5136, Phoenix, AZ, USA, 1999.

[10] C. H. Lee, T. H. Kim, and M. J. Tahk, "Design of impact angle control guidance laws via high-performance sliding mode con- trol," Proceedings of the Institution of Mechanical Engineers, Part G: Journal of Aerospace Engineering, vol. 227, no. 2, pp. 235-253, 2013.

[11] K. Zhang, S. C. Yang, K. Q. Zhang, Y. Z. Yao, and P. Chen, "Finite-time convergent sliding mode guidance law with impact angle constraint," Electronics Optics \& Control, vol. 24, no. 1, pp. 63-66+81, 2017.

[12] Z. Hou, Y. Yang, L. Liu, and Y. Wang, "Terminal sliding mode control based impact time and angle constrained guidance," Aerospace Science and Technology, vol. 93, article 105142, 2019.

[13] T. H. Kim, C. H. Lee, I. S. Jeon, and M. J. Tahk, “Augmented polynomial guidance with impact time and angle constraints," IEEE Transactions on Aerospace and Electronic Systems, vol. 49, no. 4, pp. 2806-2817, 2013.

[14] S. R. Kumar, S. Rao, and D. Ghose, "Nonsingular terminal sliding mode guidance with impact angle constraints," Journal of Guidance, Control, and Dynamics, vol. 37, no. 4, pp. 11141130, 2014.

[15] N. Harl and S. N. Balakrishnan, "Impact time and angle guidance with sliding mode control," IEEE Transactions on Control Systems Technology, vol. 20, no. 6, pp. 1436-1449, 2012.

[16] S. R. Kumar, S. Rao, and D. Ghose, "Non-singular terminal sliding mode guidance and control with terminal angle constraints for non-maneuvering targets," in 2012 12th International Workshop on Variable Structure Systems, pp. 291-296, Mumbai, Maharashtra, 2012.

[17] S. R. Kumar, S. Rao, and D. Ghose, "Sliding-mode guidance and control for all-aspect interceptors with terminal angle constraints," Journal of Guidance Control \& Dynamics, vol. 35, no. 4, pp. 1230-1246, 2012.

[18] Q. Z. Zhang, Z. B. Wang, F. Tao, and B. R. Sarker, "On-line optimization design of sliding mode guidance law with multiple constraints," Applied Mathematical Modelling, vol. 37, no. 14-15, pp. 7568-7587, 2013.

[19] P. Wu M. Yang et al., "Integrated guidance and control design for missile with terminal impact angle constraint based on sliding mode control," Journal of Systems Engineering and Electronics, vol. 21, no. 4, pp. 623-628, 2010.

[20] S. He, D. Lin, and J. Wang, "Continuous second-order sliding mode based impact angle guidance law," Aerospace Science \& Technology, vol. 41, pp. 199-208, 2015.

[21] D. Chwa and J. Y. Choi, "Adaptive nonlinear guidance law considering control loop dynamics," IEEE Transactions on Aerospace and Electronic Systems, vol. 39, no. 4, pp. 11341143, 2003.

[22] J. Song, S. Song, and H. Zhou, “Adaptive nonsingular fast terminal sliding mode guidance law with impact angle constraints," International Journal of Control, Automation and Systems, vol. 14, no. 1, pp. 99-114, 2016.

[23] J. S. Huang, H. B. Zhang, G. J. Tang, and W. M. Bao, “Adaptive integral sliding mode guidance law for intercepting maneuvering targets in the atmosphere," Journal of Astronautics, vol. 40, no. 1, pp. 55-64, 2019.

[24] B. Zhao, J. Zhou, X. D. Lu, and Y. Li, "Adaptive integral sliding mode guidance law considering terminal angle constraint," Control and Decision, vol. 32, no. 11, pp. 1966-1972, 2017.

[25] S. C. Li, R. Z. He, G. J. Tang, and P. Ao, "Profile tracking guidance law based on sliding mode control," Acta Aeronautica et Astronautica Sinica, vol. 41, Supplement 2, pp. 240 247, 2020. 
[26] Y. S. Jiang, X. L. Li, and H. T. Chen, "Basic composition and guidance process of a beam laser guidance instrument," Optics Technology, vol. 31, no. 3, pp. 354-356, 2005.

[27] F. Y. Dong, "Design of missile sliding mode guidance law based on interception geometry of three-point method," Journal of Ballistics, vol. 31, no. 2, pp. 55-59, 2019.

[28] S. P. Bhat and D. S. Bernstein, "Geometric homogeneity with applications to finite-time stability," Mathematics of Control, Signals, and Systems, vol. 17, no. 2, pp. 101-127, 2005.

[29] V. Utkin, "Variable structure systems with sliding modes," IEEE Transactions on Automatic Control, vol. 22, no. 2, pp. 212-222, 1977.

[30] S. Yu, X. Yu, B. Shirinzadeh, and Z. Man, "Continuous finitetime control for robotic manipulators with terminal sliding mode," Automatica, vol. 41, no. 11, pp. 1957-1964, 2005. 